\title{
NON-UNIQUENESS OF GIBBS MEASURE FOR MODELS WITH UNCOUNTABLE SET OF SPIN VALUES ON A CAYLEY TREE
}

\author{
YU. KH. ESHKABILOV, F. H. HAYDAROV, U. A. ROZIKOV
}

\begin{abstract}
In this paper we construct several models with nearest-neighbor interactions and with the set $[0,1]$ of spin values, on a Cayley tree of order $k \geq 2$. We prove that each of the constructed model has at least two translational-invariant Gibbs measures.
\end{abstract}

\author{
Mathematics Subject Classifications (2010). 82B05, 82B20 (primary); 60K35 \\ (secondary)
}

Key words. Cayley tree, configuration, Gibbs measures, uniqueness.

\section{INTRODUCTION}

Spin systems on lattices are a large class of systems considered in statistical mechanics. Some of them have a real physical meaning, others are studied as suitably simplified models of more complicated systems. The structure of the lattice (graph) plays an important role in investigations of spin systems. For example, in order to study the phase transition problem for a system on $Z^{d}$ and on Cayley tree there are two different methods: Pirogov-Sinai theory on $Z^{d}$, Markov random field theory and recurrent equations of this theory on Cayley tree. In [1]- [4], [7], [8]- [9], [12], [13], [14] for several models on Cayley tree, using the Markov random field theory Gibbs measures are described.

These papers are devoted to models with a finite set of spin values. Mainly were shown that these models have finitely many translation-invariant and uncountable numbers of the non-translation-invariant extreme Gibbs measures. Also for several models (see, for example, [5, 7, 9]) it were proved that there exist three periodic Gibbs measures (which are invariant with respect to normal subgroups of finite index of the group representation of the Cayley tree) and there are uncountable number of non-periodic Gibbs measures.

In [6] the Potts model with a countable set of spin values on a Cayley tree is considered and it was showed that the set of translation-invariant splitting Gibbs measures of the model contains at most one point, independently on parameters of the Potts model with countable set of spin values on the Cayley tree. This is a crucial difference from the models with a finite set of spin values, since the last ones may have more than one translation-invariant Gibbs measures.

This paper is continuation of our investigations [3, [10]. In [10] models (Hamiltonians) with nearest-neighbor interactions and with the (uncountable) set $[0,1]$ of spin values, on a Cayley tree of order $k \geq 1$ were studied.

A central problem in the theory of Gibbs measures is to describe infinite-volume (or limiting) Gibbs measures corresponding to a given Hamiltonian. In [10] we reduced the 
problem to the description of the solutions of some nonlinear integral equation. Then for $k=1$ we showed that the integral equation has a unique solution. In case $k \geq 2$ some models (with the set $[0,1]$ of spin values) which have a unique splitting Gibbs measure are constructed. In our next paper [3] it was found a sufficient condition on Hamiltonian of the model with an uncountable set of spin values under which the model has unique translation-invariant splitting Gibbs measure. But we had not any example of model (with uncountable spin values) with more than one translation-invariant Gibbs measure.

This problem is solved in this paper: we shall construct several models with nearestneighbor interactions and with the set $[0,1]$ of spin values, on a Cayley tree of order $k \geq 2$. We prove that each of the constructed model have at least two translational-invariant Gibbs measures.

\section{Preliminaries}

A Cayley tree $\Gamma^{k}=(V, L)$ of order $k \geq 1$ is an infinite homogeneous tree, i.e., a graph without cycles, with exactly $k+1$ edges incident to each vertices. Here $V$ is the set of vertices and $L$ that of edges (arcs).

Consider models where the spin takes values in the set $[0,1]$, and is assigned to the vertexes of the tree. For $A \subset V$ a configuration $\sigma_{A}$ on $A$ is an arbitrary function $\sigma_{A}: A \rightarrow[0,1]$. Denote $\Omega_{A}=[0,1]^{A}$ the set of all configurations on $A$. A configuration $\sigma$ on $V$ is then defined as a function $x \in V \mapsto \sigma(x) \in[0,1]$; the set of all configurations is $[0,1]^{V}$. The (formal) Hamiltonian of the model is :

$$
H(\sigma)=-J \sum_{\langle x, y\rangle \in L} \xi_{\sigma(x) \sigma(y)},
$$

where $J \in R \backslash\{0\}$ and $\xi:(u, v) \in[0,1]^{2} \rightarrow \xi_{u v} \in R$ is a given bounded, measurable function. As usually, $\langle x, y\rangle$ stands for nearest neighbor vertices.

Let $\lambda$ be the Lebesgue measure on $[0,1]$. On the set of all configurations on $A$ the a priori measure $\lambda_{A}$ is introduced as the $|A|$ fold product of the measure $\lambda$. Here and further on $|A|$ denotes the cardinality of $A$. We consider a standard sigma-algebra $\mathcal{B}$ of subsets of $\Omega=[0,1]^{V}$ generated by the measurable cylinder subsets. A probability measure $\mu$ on $(\Omega, \mathcal{B})$ is called a Gibbs measure (with Hamiltonian $H$ ) if it satisfies the DLR equation, namely for any $n=1,2, \ldots$ and $\sigma_{n} \in \Omega_{V_{n}}$ :

$$
\mu\left(\left\{\sigma \in \Omega:\left.\sigma\right|_{V_{n}}=\sigma_{n}\right\}\right)=\int_{\Omega} \mu(\mathrm{d} \omega) \nu_{\left.\omega\right|_{W_{n+1}}}^{V_{n}}\left(\sigma_{n}\right)
$$

where $\nu_{\left.\omega\right|_{W_{n+1}}}^{V_{n}}$ is the conditional Gibbs density

$$
\nu_{\left.\omega\right|_{W_{n+1}}}^{V_{n}}\left(\sigma_{n}\right)=\frac{1}{Z_{n}\left(\left.\omega\right|_{W_{n+1}}\right)} \exp \left(-\beta H\left(\sigma_{n} \|\left.\omega\right|_{W_{n+1}}\right)\right)
$$

and $\beta=\frac{1}{T}, T>0$ is temperature. Here and below, $W_{l}$ stands for a 'sphere' and $V_{l}$ for a 'ball' on the tree, of radius $l=1,2, \ldots$, centered at a fixed vertex $x^{0}$ (an origin):

$$
W_{l}=\left\{x \in V: d\left(x, x^{0}\right)=l\right\}, \quad V_{l}=\left\{x \in V: d\left(x, x^{0}\right) \leq l\right\}
$$


and

$$
L_{n}=\left\{\langle x, y\rangle \in L: x, y \in V_{n}\right\}
$$

distance $d(x, y), x, y \in V$, is the length of (i.e. the number of edges in) the shortest path connecting $x$ with $y . \Omega_{V_{n}}$ is the set of configurations in $V_{n}$ (and $\Omega_{W_{n}}$ that in $W_{n}$; see below). Furthermore, $\left.\sigma\right|_{V_{n}}$ and $\left.\omega\right|_{W_{n+1}}$ denote the restrictions of configurations $\sigma, \omega \in \Omega$ to $V_{n}$ and $W_{n+1}$, respectively. Next, $\sigma_{n}: x \in V_{n} \mapsto \sigma_{n}(x)$ is a configuration in $V_{n}$ and $H\left(\left.\sigma_{n}|| \omega\right|_{W_{n+1}}\right)$ is defined as the sum $H\left(\sigma_{n}\right)+U\left(\sigma_{n},\left.\omega\right|_{W_{n+1}}\right)$ where

$$
\begin{aligned}
H\left(\sigma_{n}\right) & =-J \sum_{\langle x, y\rangle \in L_{n}} \xi_{\sigma_{n}(x) \sigma_{n}(y)}, \\
U\left(\sigma_{n},\left.\omega\right|_{W_{n+1}}\right) & =-J \sum_{\langle x, y\rangle: x \in V_{n}, y \in W_{n+1}} \xi_{\sigma_{n}(x) \omega(y)} .
\end{aligned}
$$

Finally, $Z_{n}\left(\left.\omega\right|_{W_{n+1}}\right)$ stands for the partition function in $V_{n}$, with the boundary condition $\left.\omega\right|_{W_{n+1}}$ :

$$
Z_{n}\left(\left.\omega\right|_{W_{n+1}}\right)=\int_{\Omega_{V_{n}}} \exp \left(-\beta H\left(\left.\widetilde{\sigma}_{n}|| \omega\right|_{W_{n+1}}\right)\right) \lambda_{V_{n}}\left(d \widetilde{\sigma}_{n}\right) .
$$

Due to the nearest-neighbor character of the interaction, the Gibbs measure possesses a natural Markov property: for given a configuration $\omega_{n}$ on $W_{n}$, random configurations in $V_{n-1}$ (i.e., 'inside' $\left.W_{n}\right)$ and in $V \backslash V_{n+1}$ (i.e., 'outside' $W_{n}$ ) are conditionally independent.

We use a standard definition of a translation-invariant measure (see, e.g., [11]). The main object of study in this paper are translation-invariant Gibbs measures for the model (2.1) on Cayley tree. In [10] this problem of description of such measures was reduced to the description of the solutions of a nonlinear integral equation. For finite and countable sets of spin values this argument is well known (see, e.g. [1]- 6], [8], [12, [13, [14]).

Write $x<y$ if the path from $x^{0}$ to $y$ goes through $x$. Call vertex $y$ a direct successor of $x$ if $y>x$ and $x, y$ are nearest neighbors. Denote by $S(x)$ the set of direct successors of $x$. Observe that any vertex $x \neq x^{0}$ has $k$ direct successors and $x^{0}$ has $k+1$.

Let $h: x \in V \mapsto h_{x}=\left(h_{t, x}, t \in[0,1]\right) \in R^{[0,1]}$ be mapping of $x \in V \backslash\left\{x^{0}\right\}$. Given $n=1,2, \ldots$, consider the probability distribution $\mu^{(n)}$ on $\Omega_{V_{n}}$ defined by

$$
\mu^{(n)}\left(\sigma_{n}\right)=Z_{n}^{-1} \exp \left(-\beta H\left(\sigma_{n}\right)+\sum_{x \in W_{n}} h_{\sigma(x), x}\right),
$$

Here, as before, $\sigma_{n}: x \in V_{n} \mapsto \sigma(x)$ and $Z_{n}$ is the corresponding partition function:

$$
Z_{n}=\int_{\Omega_{V_{n}}} \exp \left(-\beta H\left(\widetilde{\sigma}_{n}\right)+\sum_{x \in W_{n}} h_{\widetilde{\sigma}(x), x}\right) \lambda_{V_{n}}\left(d \widetilde{\sigma}_{n}\right) .
$$

The probability distributions $\mu^{(n)}$ are compatible if for any $n \geq 1$ and $\sigma_{n-1} \in \Omega_{V_{n-1}}$ :

$$
\int_{\Omega_{W_{n}}} \mu^{(n)}\left(\sigma_{n-1} \vee \omega_{n}\right) \lambda_{W_{n}}\left(d\left(\omega_{n}\right)\right)=\mu^{(n-1)}\left(\sigma_{n-1}\right) .
$$


Here $\sigma_{n-1} \vee \omega_{n} \in \Omega_{V_{n}}$ is the concatenation of $\sigma_{n-1}$ and $\omega_{n}$. In this case there exists a unique measure $\mu$ on $\Omega_{V}$ such that, for any $n$ and $\sigma_{n} \in \Omega_{V_{n}}, \mu\left(\left\{\left.\sigma\right|_{V_{n}}=\sigma_{n}\right\}\right)=$ $\mu^{(n)}\left(\sigma_{n}\right)$.

Definition 2.1. The measure $\mu$ is called splitting Gibbs measure corresponding to Hamiltonian (2.1) and function $x \mapsto h_{x}, x \neq x^{0}$.

The following statement describes conditions on $h_{x}$ guaranteeing compatibility of the corresponding distributions $\mu^{(n)}\left(\sigma_{n}\right)$.

Proposition 2.2. [10] The probability distributions $\mu^{(n)}\left(\sigma_{n}\right), n=1,2, \ldots$, in (2.2) are compatible iff for any $x \in V \backslash\left\{x^{0}\right\}$ the following equation holds:

$$
f(t, x)=\prod_{y \in S(x)} \frac{\int_{0}^{1} \exp \left(J \beta \xi_{t u}\right) f(u, y) d u}{\int_{0}^{1} \exp \left(J \beta \xi_{0 u}\right) f(u, y) d u} .
$$

Here, and below $f(t, x)=\exp \left(h_{t, x}-h_{0, x}\right), t \in[0,1]$ and $d u=\lambda(d u)$ is the Lebesgue measure.

From Proposition 2.2 it follows that for any $h=\left\{h_{x} \in R^{[0,1]}, x \in V\right\}$ satisfying (2.5) there exists a unique Gibbs measure $\mu$ and vice versa. However, the analysis of solutions to (2.5) is not easy. This difficulty depends on the given function $\xi$.

Let $\xi_{t u}$ is a continuous function and we are going to construct functions $\xi_{t u}$ under which the equation (2.5) has at least two solutions in the class of translational-invariant functions $f(t, x)$, i.e $f(t, x)=f(t)$, for any $x \in V$. For such functions equation (2.5) can be written as

$$
f(t)=\left(\frac{\int_{0}^{1} K(t, u) f(u) d u}{\int_{0}^{1} K(0, u) f(u) d u}\right)^{k}
$$

where $K(t, u)=\exp \left(J \beta \xi_{t u}\right), f(t)>0, t, u \in[0,1]$.

We put

$$
C^{+}[0,1]=\{f \in C[0,1]: f(x) \geq 0\} .
$$

We are interested to positive continuous solutions to (2.6), i.e. such that

$f \in C_{0}^{+}[0,1]=\{f \in C[0,1]: f(x) \geq 0\} \backslash\{\theta \equiv 0\}$.

Note that equation (2.6) is not linear for any $k \geq 1$.

Define the operator $R_{k}: C_{0}^{+}[0,1] \rightarrow C_{0}^{+}[0,1]$ by

$$
\left(R_{k} f\right)(t)=\left[\frac{(W f)(t)}{(W f)(0)}\right]^{k}, k \in \mathbb{N},
$$

where $W: C[0,1] \rightarrow C[0,1]$ is linear operator, which is defined by :

$$
(W f)(t)=\int_{0}^{1} K(t, u) f(u) d u .
$$

Then the equation (2.6) can be written as 


$$
R_{k} f=f, \quad f \in C_{0}^{+}[0,1]
$$

\section{The HAmmerstein's NONLINEAR INTEGRAL EQUATION}

For every $k \in \mathbb{N}$ we consider an integral operator $H_{k}$ acting in the cone $C^{+}[0,1]$ as

$$
\left(H_{k} f\right)(t)=\int_{0}^{1} K(t, u) f^{k}(u) d u, k \in \mathbb{N} .
$$

The operator $H_{k}$ is called Hammerstein's integral operator of order $k$. Clearly that, if $k \geq 2$ then $H_{k}$ is a nonlinear operator.

Lemma 3.1. Let $k \geq 2$. The equation

$$
R_{k} f=f, \quad f \in C_{0}^{+}[0,1]
$$

has a nontrivial positive solution iff the Hammerstein's operator has a positive eigenvalue, i.e. the Hammerstein's equation

$$
H_{k} f=\lambda f, \quad f \in C^{+}[0,1]
$$

has a nonzero positive solution for some $\lambda>0$.

Proof. Necessariness. Let $f_{0} \in C_{0}^{+}[0,1]$ be a solution of the equation (3.1). We have

$$
\left(W f_{0}\right)(t)=\left(W f_{0}\right)(0) f_{0}^{\frac{1}{k}}(t)
$$

From this equality we get

$$
\left(H_{k} h\right)(t)=\lambda_{0} h(t)
$$

where $h(t)=\sqrt[k]{f_{0}(t)} \in C_{0}^{+}[0,1]$ and $\lambda_{0}=\left(W f_{0}\right)(0)$, i.e., the number $\lambda_{0}$ is the positive eigenvalue of the Hammerstein's operator $H_{k}$.

Sufficiency. Let $\lambda_{0}$ be a positive eigenvalue of the operator $H_{k}$. Then $\lambda_{0}>0$ and there exists $f_{0} \in C_{0}^{+}[0,1]$ such that

$$
H_{k} f_{0}=\lambda_{0} f_{0}
$$

Obviously, the function $f_{0}(t)$ is a strictly positive. Put

$$
f(t)=\frac{f_{0}(t)}{f_{0}(0)}, t \in[0,1]
$$

Then the number $\lambda=\lambda_{0} f_{0}^{1-k}(0)$ is an eigenvalue of $H_{k}$ and corresponding the positive eigenfunction $f(t)$ satisfies

Define

$$
H_{k} f(t)=\frac{1}{f_{0}^{k}(0)}\left(H_{k} f_{0}\right)(t)=\frac{\lambda_{0}}{f_{0}^{k}(0)} f_{0}(t)=\lambda_{0} f_{0}^{1-k}(0) f(t)=\lambda f(t)
$$

$$
h(t)=\left(\frac{f_{0}(t)}{f_{0}(0)}\right)^{k}=f^{k}(t)
$$


Then

$$
\left(R_{k} h\right)(t)=\left(\frac{(W h)(t)}{(W h)(0)}\right)^{k}=\left(\frac{\left(H_{k} f\right)(t)}{\left(H_{k} f\right)(0)}\right)^{k}=\left(\frac{\lambda_{0} f_{0}^{1-k}(0) f(t)}{\lambda_{0} f_{0}^{1-k}(0) f(0)}\right)^{k}=f^{k}(t)=h(t)
$$

Corollary 3.2. Let $k \geq 2$. If a function $f \in C_{0}^{+}[0,1]$ is an eigenfunction of $H_{k}$, then the function

$$
h(t)=\left(\frac{f(t)}{f(0)}\right)^{k}
$$

is a solution to the equation (3.1).

4. Existence of two Gibbs measures for the model (2.1): CASE $k=2$

Consider the case $k=2$ in the model (2.1) and

$$
\xi_{t, u}=\frac{1}{\beta J} \ln \left(1+\frac{14}{15} \cdot \sqrt[5]{4\left(t-\frac{1}{2}\right)\left(u-\frac{1}{2}\right)}\right), t, u \in[0,1] .
$$

Then, for the kernel $K(t, u)$ of the Hammerstein's integral operator $H_{2}$ we have

$$
K(t, u)=1+\frac{14}{15} \cdot \sqrt[5]{4\left(t-\frac{1}{2}\right)\left(u-\frac{1}{2}\right)}
$$

Proposition 4.1. The Hammerstein's operator $\mathrm{H}_{2}$ :

$$
\left(H_{2} f\right)(t)=\int_{0}^{1} K(t, u) f^{2}(u) d u
$$

in the space $C[0,1]$ has at least two strictly positive fixed points.

Proof. a) Let $f_{1}(t) \equiv 1$. Then we have

$$
\left(H_{2} f_{1}\right)(t)=1+\frac{14}{15} \cdot \sqrt[5]{4\left(t-\frac{1}{2}\right)} \cdot \int_{0}^{1}\left(u-\frac{1}{2}\right)^{\frac{1}{5}} d u=1=f_{1}(t), \quad t \in[0,1] .
$$

b) Denote

$$
f_{2}(t)=\frac{3}{4}+\sqrt{\frac{21}{5}} \cdot \frac{\sqrt[5]{2}}{4} \cdot\left(t-\frac{1}{2}\right)^{\frac{1}{5}}, t \in[0,1] .
$$

Then $f_{2} \in C[0,1]$ and the function $f_{2}(t)$ is strictly positive. Put

$$
a=\frac{14}{15} \cdot \sqrt[5]{4}, \quad b=\sqrt{\frac{21}{5}} \cdot \frac{\sqrt[5]{2}}{4} .
$$

We have

$$
H_{2} f_{2}=h_{1}(t)+h_{2}(t)+h_{3}(t)+\gamma
$$

where 


$$
\begin{gathered}
h_{1}(t)=a b^{2} \cdot \sqrt[5]{t-\frac{1}{2}} \cdot \int_{0}^{1} \sqrt[5]{\left(u-\frac{1}{2}\right)^{3}} d u \\
h_{2}(t)=\frac{3 a b}{2} \cdot \sqrt[5]{t-\frac{1}{2}} \cdot \int_{0}^{1} \sqrt[5]{\left(u-\frac{1}{2}\right)^{2}} d u \\
h_{3}(t)=\frac{9 a}{16} \cdot \sqrt[5]{t-\frac{1}{2}} \cdot \int_{0}^{1} \sqrt[5]{u-\frac{1}{2}} d u \\
\gamma=\int_{0}^{1} f_{2}^{2}(u) d u
\end{gathered}
$$

It is clear that

$$
h_{1}(t)=h_{3}(t) \equiv 0
$$

For the function $h_{2}(t)$ we obtain

$$
h_{2}(t)=\frac{3 a b}{2} \cdot \sqrt[5]{t-\frac{1}{2}} \cdot \int_{-1 / 2}^{1 / 2} u^{\frac{2}{5}} d u=\frac{15 a b}{14 \sqrt[5]{4}} \cdot \sqrt[5]{t-\frac{1}{2}} .
$$

Observe that

$$
\gamma=\frac{5 b^{2}}{7 \sqrt[5]{4}}+\frac{9}{16}
$$

Consequently we have

$$
\left(H_{2} f_{2}\right)(t)=h_{2}(t)+\gamma=\frac{15 a b}{14 \sqrt[5]{4}} \cdot \sqrt[5]{t-\frac{1}{2}}+\frac{5 b^{2}}{7 \sqrt[5]{4}}+\frac{9}{16}=\sqrt{\frac{21}{5}} \cdot \frac{\sqrt[5]{2}}{4} \cdot \sqrt[5]{t-\frac{1}{2}}+\frac{3}{4}=f_{2}(t)
$$

Denote by $\mu_{1}$ and $\mu_{2}$ the translation-invariant Gibbs measures which by Proposition 2.2 correspond to solutions $f_{1}(t)=1$ and $f_{2}(t)=\frac{3}{4}+\sqrt{\frac{21}{5}} \cdot \frac{\sqrt[5]{2}}{4} \cdot\left(t-\frac{1}{2}\right)^{\frac{1}{5}}$.

Thus we have proved the following

Theorem 4.2. The model

$$
H(\sigma)=-\frac{1}{\beta} \sum_{\substack{<x, y>\\ x, y \in V}} \ln \left(1+\frac{14}{15} \cdot \sqrt[5]{4\left(\sigma(x)-\frac{1}{2}\right)\left(\sigma(y)-\frac{1}{2}\right)}\right), \sigma \in \Omega_{V}
$$

on the Cayley tree $\Gamma^{2}$ has at least two translation-invariant Gibbs measures $\mu_{1}, \mu_{2}$. 
5. Existence of two Gibbs measures for the model (2.1): Case $k=3$

Now we shall consider the case $k=3$ and

$$
\xi_{t, u}=\frac{1}{\beta J} \ln \left(1+\frac{1}{2} \cdot \sqrt[7]{4\left(t-\frac{1}{2}\right)\left(u-\frac{1}{2}\right)}\right), t, u \in[0,1] .
$$

Then, for the kernel $K(t, u)$ of the operator $H_{3}$ we have

$$
K(t, u)=1+\frac{1}{2} \sqrt[7]{4\left(t-\frac{1}{2}\right)\left(u-\frac{1}{2}\right)} .
$$

Proposition 5.1. The operator $H_{3}$ :

$$
\left(H_{3} f\right)(t)=\int_{0}^{1}\left(1+\frac{1}{2} \cdot \sqrt[7]{4\left(t-\frac{1}{2}\right)\left(u-\frac{1}{2}\right)}\right) f^{3}(u) d u
$$

in the space $C[0,1]$ has at least two strictly positive fixed points.

Proof. a) Let $f_{1}(t) \equiv 1$. Then

$$
\left(H_{3} f_{1}\right)(t)=1+\frac{1}{2} \cdot \sqrt[7]{4\left(t-\frac{1}{2}\right)} \cdot \int_{-\frac{1}{2}}^{\frac{1}{2}} u^{\frac{1}{7}} d u=1=f_{1}(t), \quad t \in[0,1] .
$$

b) We define the function $f_{2}$ :

$$
f_{2}(t)=\frac{1}{2}\left(\sqrt{\frac{57}{17}}+\sqrt{\frac{33}{119}} \cdot \sqrt[7]{2\left(t-\frac{1}{2}\right)}\right), t \in[0,1] .
$$

Then $f_{2} \in C[0,1]$ and the function $f_{2}(t)$ is strictly positive. Put

$$
a=\frac{1}{2} \sqrt{\frac{57}{17}}, b=\frac{1}{2} \sqrt{\frac{33}{119}} .
$$

We have

$$
\left(H_{3} f_{2}\right)(t)=h_{1}(t)+h_{2}(t)+h_{3}(t)+h_{4}(t)+\gamma,
$$

where

$$
\begin{gathered}
h_{1}(t)=\frac{a^{3}}{2} \varphi(t) \cdot \int_{0}^{1} \sqrt[7]{u-\frac{1}{2}} d u \\
h_{2}(t)=\frac{3 a^{2} b}{2} \cdot \sqrt[7]{2} \varphi(t) \cdot \int_{0}^{1} \sqrt[7]{\left(u-\frac{1}{2}\right)^{2}} d u \\
h_{3}(t)=\frac{3 a b^{2}}{2} \cdot \sqrt[7]{4} \varphi(t) \cdot \int_{0}^{1} \sqrt[7]{\left(u-\frac{1}{2}\right)^{3}} d u \\
h_{4}(t)=\frac{b^{3}}{2} \cdot \sqrt[7]{8} \varphi(t) \cdot \int_{0}^{1} \sqrt[7]{\left(u-\frac{1}{2}\right)^{4}} d u
\end{gathered}
$$




$$
\gamma=\int_{0}^{1} f_{2}^{3}(u) d u
$$

Here $\varphi(t)=\sqrt[7]{4\left(t-\frac{1}{2}\right)}, t \in[0,1]$

It is clear that

$$
h_{1}(t)=h_{3}(t) \equiv 0
$$

For the functions $h_{2}(t)$ and $h_{4}(t)$ we obtain, that

$$
\begin{aligned}
& h_{2}(t)=\frac{3 a^{2} b \sqrt[7]{2}}{2} \cdot \varphi(t) \int_{-\frac{1}{2}}^{\frac{1}{2}} u^{\frac{2}{7}} d u=\frac{7 a^{2} b}{6 \sqrt[7]{2}} \cdot \varphi(t), \\
& h_{4}(t)=\frac{b^{3} \sqrt[7]{8}}{2} \cdot \varphi(t) \int_{-\frac{1}{2}}^{\frac{1}{2}} u^{\frac{4}{7}} d u=\frac{7 b^{3}}{22 \sqrt[7]{2}} \cdot \varphi(t) .
\end{aligned}
$$

Observe that

$$
\gamma=a^{3}+3 a b^{2} \sqrt[7]{4} \cdot \int_{-\frac{1}{2}}^{\frac{1}{2}} u^{\frac{2}{7}} d u=a^{3}+\frac{7 a b^{2}}{3}=a .
$$

Consequently, we have

$$
H_{3} f_{2}=h_{2}+h_{4}+a=a+\frac{7 b}{2 \sqrt[7]{2}}\left(\frac{a^{2}}{3}+\frac{b^{2}}{11}\right) \varphi(t)=a+\frac{b}{\sqrt[7]{2}} \varphi(t)=f_{2}(t) .
$$

From Proposition 5.1, Lemma 3.1 and Proposition 2.2 we get

Theorem 5.2. The model

$$
H(\sigma)=-\frac{1}{\beta} \sum_{\substack{<x, y>\\ x, y \in V}} \ln \left(1+\frac{1}{2} \sqrt[7]{4\left(\sigma(x)-\frac{1}{2}\right)\left(\sigma(y)-\frac{1}{2}\right)}\right), \sigma \in \Omega_{V}
$$

on the Cayley tree $\Gamma^{3}$ has at least two translation-invariant Gibbs measures.

6. Existence of two Gibbs measures for the model (2.1): Case $k \geq 4$

Let $k \in \mathbb{N}$ and $k \geq 2$. We consider sequences of continuous functions $P_{n}(x)(n \in \mathbb{N})$ and $Q_{m}(x)(m \in \mathbb{N}, m>k)$ defined by

$$
\begin{gathered}
P_{n}(x) \equiv P_{n, k}(x)=\left(1+\frac{x^{n-1}}{2}\right)^{k+1}-\left(1-\frac{x^{n-1}}{2}\right)^{k+1}, x \in \mathbb{R}, \\
Q_{m}(x) \equiv Q_{m, k}(x)=(k+1) x^{m-k}, m>k, x \in \mathbb{R} .
\end{gathered}
$$

Proposition 6.1. Let $k \geq 2$. Then

$$
P_{n}(1)>Q_{n}(1)
$$

for any $n \in \mathbb{N}, n>k$. 
Proof. Let $k \geq 2$ and $n>k$. We have

$$
P_{n}(1)=\mu_{k}=\frac{3^{k+1}-1}{2^{k+1}}, Q_{n}=\eta_{k}=k+1 .
$$

In the case $k=2$ we obtain, that

$$
P_{n}(1)=\frac{13}{4}>Q_{n}(1)=3 .
$$

We now suppose, that the inequality (6.1) holds for $k=m>2$. Then we show that the inequality (6.1) also is true for $k=m+1$.

Obviously, that

$\mu_{m+1}=\frac{3^{(m+1)+1}-1}{2^{(m+1)+1}}>\frac{3^{(m+1)+1}-3}{2^{m+1} \cdot 2}=\frac{3^{m+1}-1}{2^{m+1}} \cdot \frac{3}{2}=\mu_{m} \cdot \frac{3}{2}>(m+1) \cdot \frac{3}{2}>m+2=\eta_{m+1}$,

i.e. $\mu_{m+1}>\eta_{m+1}$. Thus we get

$$
P_{n}(1)>Q_{n}(1)
$$

for any $k \geq 2$ and $n>k$.

Proposition 6.2. Let $k \geq 2$. The equation

$$
\left(1+\frac{x}{2}\right)^{k+1}-\left(1-\frac{x}{2}\right)^{k+1}-(k+1) x=0, \quad x \geq 0
$$

has a unique solution $x=0$.

Proof. Let $k \geq 2$. Define the continuous function $\varphi(x)$ :

$$
\varphi(x)=\left(1+\frac{x}{2}\right)^{k+1}-\left(1-\frac{x}{2}\right)^{k+1}-(k+1) x, \quad x \in[0, \infty) .
$$

We have

$$
\varphi^{\prime}(x)=(k+1)\left(\frac{1}{2}\left(1+\frac{x}{2}\right)^{k}+\frac{1}{2}\left(1-\frac{x}{2}\right)^{k}-1\right) .
$$

However,

$$
\left(1+\frac{x}{2}\right)^{k}+\left(1-\frac{x}{2}\right)^{k}>2, \text { for all } x \in(0, \infty)
$$

Consequently, we have $\varphi^{\prime}(x)>0$ for all $x \in(0, \infty)$, i.e. the function $\varphi(x)$ is an increasing on $[0, \infty)$. So, the zero is a unique solution of the equation (6.2).

Proposition 6.3. Let $k \geq 2$. Then for each $n \in \mathbb{N}, n>k$ the equation

$$
P_{n}(x)-Q_{n}(x)=0
$$

has at least one solution $\xi=\xi(k ; n)$ in $(0,1)$. 
Proof. Let $k \geq 2$ and $n>k$. We have

$$
\begin{gathered}
\lim _{x \rightarrow 0+} \frac{P_{n}(x)}{Q_{n}(x)}=\frac{1}{k+1} \lim _{x \rightarrow 0+} \frac{\left(1+\frac{x^{n-1}}{2}\right)^{k+1}-\left(1-\frac{x^{n-1}}{2}\right)^{k+1}}{x^{n-k}}= \\
=\frac{1}{k+1} \lim _{x \rightarrow 0+} \frac{\left(\left(1+\frac{x^{n-1}}{2}\right)-\left(1-\frac{x^{n-1}}{2}\right)\right) \sum_{j=0}^{k}\left(1+\frac{x^{n-1}}{2}\right)^{k-j}\left(1-\frac{x^{n-1}}{2}\right)^{j}}{x^{n-k}}= \\
=\frac{1}{k+1} \lim _{x \rightarrow 0+} x^{k-1} \cdot \sum_{j=o}^{k}\left(1+\frac{x^{n-1}}{2}\right)^{k-j}\left(1-\frac{x^{n-1}}{2}\right)^{j}=0 .
\end{gathered}
$$

Since the functions $P_{n}(x)$ and $Q_{n}(x)$ are continuous, the exists a number $\delta>0$ such that

$$
P_{n}(x)<Q_{n}(x) \text { for all } x \in(0, \delta) .
$$

However $P_{n}(0)=Q_{n}(0)=0$ and by Proposition 6.1 we have $P_{n}(1)>Q_{n}(1)$. Consequently, there exists a number $\xi=\xi(k ; n) \in(0,1)$ such that $P_{n}(\xi(k ; n))=Q_{n}(\xi(k ; n))=$ 0 .

Let $k \geq 2$ be a fixed number and suppose that $\{\xi(k ; n)\}_{n>k} \subset(0,1)$ - some set of solutions of the following system of equations:

$$
P_{n}(x)-Q_{n}(x)=0, n \in \mathbb{N}, n>k .
$$

We have $0<\xi(k ; n)<1$ for all $n \in \mathbb{N}, n>k$. Consequently $0<\xi(k ; n)^{n-1}<1$ for all $n>k$. Then there exists a upper limit of the sequence $\xi(k ; n)^{n-1}, n>k$, i.e. there exists a subsequence $\alpha_{p}=\xi\left(k ; n_{p}\right)^{n_{p}-1}, \quad p \in \mathbb{N}$ of the sequence $\xi(k ; n)^{n-1}, n>k$ such that

$$
\alpha=\lim _{n \rightarrow \infty} \sup \xi(k ; n)^{n-1}=\lim _{p \rightarrow \infty} \xi\left(k ; n_{p}\right)^{n_{p}-1}=\lim _{p \rightarrow \infty} \alpha_{p} .
$$

Obviously, that $0 \leq \alpha \leq 1$. Define the sequence $\beta_{p}, p \in \mathbb{N}$ by

$$
\beta_{p}=\xi\left(k ; n_{p}\right), \quad p \in \mathbb{N} .
$$

Then

$$
\alpha_{p}=\beta_{p}^{n_{p}-1}, \quad p \in \mathbb{N} \text {. }
$$

Lemma 6.4. $\alpha=\lim _{p \rightarrow \infty} \alpha_{p}=0$.

Proof. a)Assume $\alpha=1$. Put

$$
\beta=\lim _{p \rightarrow \infty} \sup \xi\left(k ; n_{p}\right)=\lim _{p \rightarrow \infty} \sup \beta_{p} .
$$

Then, there exists a subsequence $\left\{\beta_{p_{q}}\right\}_{q \in \mathbb{N}} \subset\left\{\beta_{p}\right\}_{p \in \mathbb{N}}$ such that

$$
\lim _{q \rightarrow \infty} \beta_{p_{q}}=\beta \text {. }
$$


We have $0 \leq \beta \leq 1$. If $0 \leq \beta<1$, there exists $q_{0} \in \mathbb{N}$ such that $\beta_{p_{q}}<\frac{1+\beta}{2}$ for all $q>q_{0}$. From that

$$
0 \leq \alpha_{p_{q}} \leq\left(\frac{1+\beta}{2}\right)^{n_{p_{q}}-1}, q \in \mathbb{N}, q>q_{0} .
$$

Therefore $\alpha=\lim _{q \rightarrow \infty} \alpha_{p_{q}}=0$. The last equality is a contradiction to the assumption $\alpha=1$. However, we obtain that $\beta=1$. Then from the equality

$$
P_{n_{p_{q}}}\left(\xi\left(k ; n_{p_{q}}\right)\right)=Q_{n_{p_{q}}}\left(\xi\left(k ; n_{p_{q}}\right)\right), q \in \mathbb{N}
$$

as $q \rightarrow \infty$ we observe that

$$
\left(1+\frac{1}{2}\right)^{k+1}-\left(1-\frac{1}{2}\right)^{k+1}=k+1
$$

i.e.

$$
P_{m}(1)=Q_{m}(1), \quad m>k .
$$

The last equality is a contradiction to the assertion of Proposition 6.1. Thus, we have proved that $\alpha \neq 1$.

b) Assume that $0<\alpha<1$. In the case $0 \leq \beta<1$ we get $\alpha=0$. So $\beta=1$. Then from (6.4) as $q \rightarrow \infty$ we get

$$
\left(1+\frac{\alpha}{2}\right)^{k+1}-\left(1-\frac{\alpha}{2}\right)^{k+1}=(k+1) \alpha
$$

The last equality is contradict to the assertion of Proposition 6.2. Thus, we have proved that $\alpha \notin(0,1)$. Consequently, $\alpha=0$.

Corollary 6.5. $\lim _{p \rightarrow \infty} \beta_{p}=1$.

Proof. From the equality (6.4) we get

Hence by Lemma 6.4 it follows that

$$
\beta_{p}=\xi\left(k ; n_{p}\right)=\sqrt[k-1]{\frac{k+1}{\sum_{j=0}^{k}\left(1+\frac{\alpha_{p}}{2}\right)^{k-j}\left(1-\frac{\alpha_{p}}{2}\right)^{j}}}, p \in \mathbb{N} .
$$

$$
\lim _{p \rightarrow \infty} \beta_{p}=1
$$

Define the sequence $C_{n}, \quad n>k \geq 2$ :

$$
C_{n}=C_{n}(k)=\frac{\xi(k ; n)^{3 n-k-2}}{\frac{1}{2+k} \cdot\left[\left(1+\frac{\xi(k ; n)^{n-1}}{2}\right)^{k+2}-\left(1-\frac{\xi(k ; n)^{n-1}}{2}\right)^{k+2}\right]-\xi(k ; n)^{n-k}},
$$

where $\xi(k ; n) \in(0,1)$ is an arbitrary solution to the equation (6.3).

Put

$$
\gamma_{p}=\gamma_{p}(k)=C_{n_{p}}(k), \quad p \in \mathbb{N}
$$


Lemma 6.6. For every $k \in \mathbb{N}, k \geq 2$ the following equality holds

$$
\lim _{p \rightarrow \infty} \gamma_{p}(k)=\frac{12}{k} \text {. }
$$

Proof. We have

$$
\begin{gathered}
\gamma_{p}=\frac{\alpha_{p}^{3} \cdot \beta_{p}^{1-k}}{\frac{1}{k+2} \cdot\left(\left(1+\frac{\alpha_{p}}{2}\right)^{k+2}-\left(1-\frac{\alpha_{p}}{2}\right)^{k+2}\right)-\xi\left(k ; n_{p}\right)^{n_{p}-k}}= \\
=\frac{\alpha_{p}^{3} \cdot \beta_{p}^{1-k}}{\frac{1}{k+2} \cdot\left(\left(1+\frac{\alpha_{p}}{2}\right)^{k+2}-\left(1-\frac{\alpha_{p}}{2}\right)^{k+2}\right)-\frac{1}{k+1} \cdot\left(\left(1+\frac{\alpha_{p}}{2}\right)^{k+1}-\left(1-\frac{\alpha_{p}}{2}\right)^{k+1}\right)} .
\end{gathered}
$$

However

$$
\begin{gathered}
\left(1+\frac{\alpha_{p}}{2}\right)^{k+2}-\left(1-\frac{\alpha_{p}}{2}\right)^{k+2}=\sum_{j=0}^{k+2} C_{k+2}^{j} \cdot\left(\frac{\alpha_{p}}{2}\right)^{j}-\sum_{j=0}^{k+2} C_{k+2}^{j} \cdot\left(-\frac{\alpha_{p}}{2}\right)^{j}= \\
=2 C_{k+2}^{1} \cdot \frac{\alpha_{p}}{2}+2 C_{k+2}^{3} \cdot \frac{\alpha_{p}^{3}}{2^{3}}+\ldots+2 C_{k+2}^{m_{1}} \cdot \frac{\alpha_{p}^{m_{1}}}{2^{m_{1}}}
\end{gathered}
$$

where

$$
m_{1} \equiv m_{1}(k)= \begin{cases}k+2, & \text { if } k \text { is odd } \\ k+1, & \text { if } k \text { is even }\end{cases}
$$

Analogously we have

$$
\left(1+\frac{\alpha_{p}}{2}\right)^{k+1}-\left(1-\frac{\alpha_{p}}{2}\right)^{k+1}=2 C_{k+1}^{1} \cdot \frac{\alpha_{p}}{2}+2 C_{k+1}^{3} \cdot \frac{\alpha_{p}^{3}}{2^{3}}+\ldots+2 C_{k+1}^{m_{2}} \cdot \frac{\alpha_{p}^{m_{2}}}{2^{m_{2}}},
$$

where

$$
m_{2} \equiv m_{2}(k)=\left\{\begin{array}{cl}
k+1, & \text { if } k \text { is even } \\
k, & \text { if } k \text { is odd }
\end{array}\right.
$$

i.e. $m_{2}=2 m_{0}-1, m_{0} \in \mathbb{N}$.

Therefore

$$
\begin{gathered}
\frac{1}{k+2} \cdot\left(\left(1+\frac{\alpha_{p}}{2}\right)^{k+2}-\left(1-\frac{\alpha_{p}}{2}\right)^{k+2}\right)-\frac{1}{k+1} \cdot\left(\left(1+\frac{\alpha_{p}}{2}\right)^{k+1}-\left(1-\frac{\alpha_{p}}{2}\right)^{k+1}\right)= \\
=\sum_{j=2}^{m_{0}} a_{j} \alpha_{p}^{2 j-1}+a_{m_{0}+1} \alpha_{p}^{2 m_{0}+1}=\alpha_{p}^{3}\left(a_{2}+a_{3} \alpha_{p}^{2}+a_{4} \alpha_{p}^{4}+\ldots+a_{m_{0}+1} \alpha_{p}^{2\left(m_{0}-1\right)}\right),
\end{gathered}
$$

where

$$
\begin{gathered}
a_{j}=\frac{2}{2^{2 j-1}} \cdot\left(\frac{C_{k+2}^{2 j-1}}{k+2}-\frac{C_{k+1}^{2 j-1}}{k+1}\right), j=2,3, \ldots, \\
a_{m_{0}+1}=\left\{\begin{array}{cl}
0 & \text { if } m_{1}=m_{2}, \\
\frac{1}{2^{2 m_{0}}} \cdot \frac{C_{k+2}^{2 m_{0}+1}}{k+2} & \text { if } m_{2}<m_{1} .
\end{array}\right.
\end{gathered}
$$


Obviously that

$$
a_{2}=\frac{k}{12}
$$

Thus we get

$$
\gamma_{p}=\frac{\beta_{p}^{1-k}}{\frac{k}{12}+a_{3} \alpha_{p}^{2}+a_{4} \alpha_{p}^{4}+\ldots+a_{m_{0}+1} \alpha_{p}^{2\left(m_{0}-1\right)}}, \quad p \in \mathbb{N} .
$$

Hence by Corollary 6.5 it follows that

$$
\lim _{p \rightarrow \infty} \gamma_{p}=\frac{12}{k} .
$$

Corollary 6.7. If $k \geq 4$ then $0<\lim _{p \rightarrow \infty} \gamma_{p} \leq 3$.

For each $k \geq 4$ we define the set $\mathbb{N}_{0}(k)$ :

$$
\mathbb{N}_{0}(k)=\left\{p \in \mathbb{N}:\left|\gamma_{p}(k)\right|<4\right\} .
$$

Note that, the set $\mathbb{N}_{0}(k)$ is a countable subset in the set of all natural numbers. For each $p \in \mathbb{N}_{0}(k), \quad(k \geq 4)$ we define the continuous function $K_{p}(t, u ; k)$ on $[0,1]^{2}$ by

$$
K_{p}(t, u ; k)=1+\gamma_{p}(k)\left(t-\frac{1}{2}\right)\left(u-\frac{1}{2}\right), t, u \in[0,1] .
$$

By the inequality $\left|\gamma_{p}(k)\right|<4$ it follows that, the function $K_{p}(t, u ; k)$ is strictly positive.

Theorem 6.8. Let $k \geq 4$. For each $p \in \mathbb{N}_{0}(k)$ the Hammerstein's equation

$$
\int_{0}^{1} K_{p}(t, u ; k) f^{k}(u) d u=f(t)
$$

in the $C[0,1]$ has at least two positive solutions.

Proof. Obviously, that the function $f_{0}(t) \equiv 1$ is a solution of the equation (6.6). Define the strictly positive continuous function $f_{1}(t)$ on $[0,1]$ by

$$
f_{1}(t)=\xi\left(k ; n_{p}\right)+\xi\left(k ; n_{p}\right)^{n_{p}}\left(t-\frac{1}{2}\right), t \in[0,1] .
$$

We shall prove that the function $f_{1}(t)$ also is a solution of the Hammerstein's equation (6.6) :

$$
\begin{gathered}
\int_{0}^{1} K_{p}(t, u ; k) f_{1}^{k}(u) d u=\int_{0}^{1}\left(1+\gamma_{p}(k)\left(t-\frac{1}{2}\right)\left(u-\frac{1}{2}\right)\right) \times \\
\times\left(\xi\left(k ; n_{p}\right)+\xi\left(k ; n_{p}\right)^{n_{p}}\left(u-\frac{1}{2}\right)\right)^{k} d u=\int_{-1 / 2}^{1 / 2}\left(1+\gamma_{p}(k)\left(t-\frac{1}{2}\right) u\right)\left(\beta_{p}+\beta_{p}^{n_{p}} u\right)^{k} d u= \\
=\int_{-1 / 2}^{1 / 2}\left(\beta_{p}+\beta_{p}^{n_{p}} u\right)^{k} d u+\gamma_{p}(k)\left(t-\frac{1}{2}\right) \int_{-1 / 2}^{1 / 2} u\left(\beta_{p}+\beta_{p}^{n_{p}} u\right)^{k} d u=
\end{gathered}
$$




$$
\begin{aligned}
& =\frac{\beta_{p}^{k}}{\beta_{p}^{n_{p}-1}} \int_{-1 / 2}^{1 / 2}\left(1+\beta_{p}^{n_{p}-1} u\right)^{k} d\left(1+\beta_{p}^{n_{p}-1} u\right)+\gamma_{p}(k)\left(t-\frac{1}{2}\right) \times \\
& \quad \times \frac{\beta_{p}^{k}}{\beta_{p}^{n_{p}-1}} \int_{-1 / 2}^{1 / 2} u\left(1+\beta_{p}^{n_{p}-1} u\right) d\left(1+\beta_{p}^{n_{p}-1} u\right)=\left.\frac{\beta_{p}^{k}}{\alpha_{p}} \cdot \frac{1}{k+1}\left(1+\alpha_{p} u\right)^{k+1}\right|_{-1 / 2} ^{1 / 2}+ \\
& \quad+\frac{\gamma_{p}(k) \beta_{p}^{k}}{\alpha_{p}^{2}}\left(t-\frac{1}{2}\right) \int_{-1 / 2}^{1 / 2}\left(\left(1+\alpha_{p} u\right)^{k+1}-\left(1+\alpha_{p} u\right)^{k}\right) d\left(1+\alpha_{p} u\right)= \\
& =\frac{\beta_{p}^{k}}{\alpha_{p}} \cdot \frac{1}{k+1}\left(\left(1+\frac{\alpha_{p}}{2}\right)^{k+1}-\left(1-\frac{\alpha_{p}}{2}\right)^{k+1}\right)+\frac{\gamma_{p}(k) \beta_{p}^{k}}{\alpha_{p}^{2}} \times\left(t-\frac{1}{2}\right) \cdot\left(\left.\frac{1}{k+2}\left(1+\alpha_{p} u\right)^{k+2}\right|_{-1 / 2} ^{1 / 2}-\right. \\
& \left.\left.\quad \frac{1}{k+1}\left(1+\alpha_{p} u\right)^{k+1}\right|_{-1 / 2} ^{1 / 2}\right)=\frac{\beta_{p}^{k}}{\alpha_{p}} \cdot \frac{1}{k+1} \cdot(k+1) \beta_{p}^{n_{p}-k}+\frac{\gamma_{p}(k) \beta_{p}^{k}}{\alpha_{p}^{2}} \cdot\left(t-\frac{1}{2}\right) \times \\
& \left.=\frac{1}{k+2}\left(\left(1+\frac{\alpha_{p}}{2}\right)^{k+2}-\left(1-\frac{\alpha_{p}}{2}\right)^{k+2}\right)-\frac{1}{k+1}\left(\left(1+\frac{\alpha_{p}}{2}\right)^{k+1}-\left(1-\frac{\alpha_{p}}{2}\right)^{k+1}\right)\right]= \\
& =\frac{\beta_{p}^{n_{p}}}{\alpha_{p}}+\frac{\gamma_{p}(k) \beta_{p}^{k}}{\alpha_{p}^{2}} \cdot\left(t-\frac{1}{2}\right) \cdot\left[\frac{1}{k+2}\left(\left(1+\frac{\alpha_{p}}{2}\right)^{k+2}-\left(1-\frac{\alpha_{p}}{2}\right)^{k+2}\right)-\frac{1}{k+1}(k+1) \beta_{p}^{n_{p}-k}\right]= \\
& =\beta_{p}+\frac{\gamma_{p}(k) \beta_{p}^{k}}{\alpha_{p}^{2}} \cdot\left(t-\frac{1}{2}\right)\left(\frac{1}{k+2}\left(\left(1+\frac{\alpha_{p}}{2}\right)^{k+2}-\left(1-\frac{\alpha_{p}}{2}\right)^{k+2}\right)-\beta_{p}^{n_{p}-k}\right)=
\end{aligned}
$$

From Theorem 6.8, Lemma 3.1 and Proposition 2.2 we get the following theorem.

Theorem 6.9. Let $k \geq 4$ and $p \in \mathbb{N}_{0}(k)$. The model

$$
H(\sigma)=-\frac{1}{\beta} \sum_{\substack{x, y>\\ x, y \in V}} \ln K_{p}(\sigma(x), \sigma(y) ; k), \quad \sigma \in \Omega_{V}
$$

on the Cayley tree $\Gamma^{k}$ has at least two translations-invariant Gibbs measures.

\section{ACKNOWLEDGEMENTS}

U. Rozikov thanks Institut des Hautes Études Scientifiques (IHES), Bures-sur-Yvette, France for support of his visit to IHES and IMU/CDC-program for a (travel) support. 


\section{REFERENCES}

[1] Bleher, P.M. and Ganikhodjaev N.N.: On pure phases of the Ising model on the Bethe lattice. Theor. Probab. Appl. 35 (1990), 216-227.

[2] Bleher, P.M., Ruiz, J. and Zagrebnov V.A.: On the purity of the limiting Gibbs state for the Ising model on the Bethe lattice. Journ. Statist. Phys. 79 (1995), 473-482.

[3] Eshkabilov Yu.Kh, Haydarov F.H., Rozikov U.A.: Uniqueness of Gibbs Measure for Models With Uncountable Set of Spin Values on a Cayley Tree. Arxiv:math.FA.

[4] Ganikhodjaev, N.N.: On pure phases of the ferromagnet Potts with three states on the Bethe lattice of order two. Theor. Math. Phys. 85 (1990), 163-175.

[5] Ganikhodjaev, N.N. and Rozikov, U.A. Description of periodic extreme Gibbs measures of some lattice model on the Cayley tree. Theor. and Math. Phys. 111 (1997), 480-486.

[6] Ganikhodjaev, N.N. and Rozikov, U.A. : The Potts model with countable set of spin values on a Cayley Tree. Letters Math. Phys. 75 (2006), 99-109.

[7] Ganikhodjaev, N.N. and Rozikov, U.A. On Ising model with four competing interactions on Cayley tree. Math. Phys. Anal. Geom. 12 (2009), 141-156.

[8] Preston, C.: Gibbs states on countable sets (Cambridge University Press, London 1974).

[9] Rozikov, U.A. Partition structures of the Cayley tree and applications for describing periodic Gibbs distributions. Theor. and Math. Phys. 112 (1997), 929-933.

[10] Rozikov, U.A. and Eshkabilov, Yu.Kh.: On models with uncountable set of spin values on a Cayley tree: Integral equations. Math. Phys. Anal. Geom. 13 (2010), 275-286.

[11] Sinai,Ya.G.: Theory of phase transitions: Rigorous Results (Pergamon, Oxford, 1982).

[12] Spitzer, F.: Markov random fields on an infinite tree, Ann. Prob. 3 (1975), 387-398.

[13] Suhov, Y.M. and Rozikov, U.A.: A hard - core model on a Cayley tree: an example of a loss network, Queueing Syst. 46 (2004), 197-212.

[14] Zachary, S.: Countable state space Markov random fields and Markov chains on trees, Ann. Prob. 11 (1983), 894-903.

Yu. Kh. Eshkabilov, National University of Uzbekistan, Tashkent, Uzbekistan.

E-mail address: yusup62@mail.ru

F. H. Haydarov, National University of Uzbekistan, Tashkent, Uzbekistan.

E-mail address: haydarov_imc@mail.ru

U. A. Rozikov, Institute of mathematics and information technologies, Tashkent, UzBeKISTAN.

E-mail address: rozikovu@yandex.ru 